\title{
A Model of Prefrontal Cortex Dopaminergic Modulation during the Delayed Alternation Task
}

\author{
Jean-Claude Dreher $^{1,2}$, Emmanuel Guigon ${ }^{2}$, and Yves Burnod ${ }^{2}$
}

\begin{abstract}
Working memory performance is modulated by the level of dopamine (DA) D1 receptors stimulation in the prefrontal cortex (PFC). This modulation is exerted at different time scales. Injection of D1 agonists/antagonists exerts a longlasting influence (several minutes or hours) on PFC pyramidal neurons. In contrast, during performance of a cognitive task, the duration of the postsynaptic effect of phasic DA release is short lasting. The functional relationships of these two time scales of DA modulation remain poorly understood. Here we propose a model that combines these two time scales of DA modulation on a prefrontal neural network. The model links the cellular and behavioral levels during performance of the delayed alternation task. The network, which represents the activity of deep-layer pyramidal neurons with intrinsic neuronal properties, exhibits two stable states of activity that can be
\end{abstract}

\section{INTRODUCTION}

Converging evidence from single-cell recordings and lesional studies in animals indicates that the prefrontal cortex (PFC) plays a pivotal role in mediating working memory, the ability for retaining and manipulating information temporarily (Fuster, 2001; Goldman-Rakic, 1995; Funahashi, Bruce, \& Goldman-Rakic, 1989). A common task to test working memory in animals is the delayed alternation task, which consists of alternating two responses (right and left) separated by a delay period. This task is frequently used as a sensitive indicator of PFC dysfunction because deficit of its performance is observed both in monkeys with PFC lesions (Fuster, 1995; Jacobsen, 1935) and in rats with medial PFC lesions (Delatour \& Gisquet-Verrier, 1996; Wortwein, Mogensen, \& Divac, 1994; Brito \& Brito, 1990). During the delayed alternation task, some PFC neurons are activated during the delay period, others during the delay and movement execution, and others during the movement only (Carlson, Rama, Tanila, Linnankoski, \& Mansikka, 1997; Niki, 1974b). Of particular importance for retention of the previous response and preparation

${ }^{1}$ Current address: NIMH, Bethesda, MD, ${ }^{2}$ INSERM U483, University of Paris VI Jussieu switched on and off by excitatory inputs from long-distance cortical areas arriving in superficial layers. These stable states allow PFC neurons to maintain representations during the delay period. The role of an increase of DA receptors stimulation is to restrict inputs arriving on the prefrontal network. The model explains how the level of working memory performance follows an inverted U-shape with an increased stimulation of DA D1 receptors. The model predicts that (1) D1 receptor agonists increase perseverations, (2) D1 antagonists increase distractability, and (3) the duration of the postsynaptic effect of phasic DA release in the PFC is adjusted to the delay period of the task. These results show how the precise duration of the postsynaptic effect of phasic DA release influences behavioral performance during a simple cognitive task.

of the following response are the neurons exhibiting sustained activity during the delay (Miller, Erickson, \& Desimone, 1996a; Alexander, 1982; Fuster, 1973). A majority of the neurons responding during the delay period of the delayed alternation task are spatially selective, that is, their discharge frequency is different during the delay between right-sided and left-sided trials (Carlson et al., 1997; Niki, 1974a).

The origins of sustained activities of pyramidal neurons have either been attributed to recurrent connections and/or to intrinsic neuronal mechanisms (see Methods) (Camperi \& Wang, 1998; Delord, Klaassen, Burnod, Costalat, \& Guigon, 1997; Marder, Abbott, Turrigiano, \& Golowasch, 1996). Our model combines these two properties and distinguishes basal dendrites of PFC deep-layer pyramidal neurons, receiving recurrent connections from neighboring cortical columns, from apical dendrites, receiving long-distance inputs from long-range cortical areas (Figure 1). The latter transitory excitatory inputs, representing go-signals or noise (distracting stimuli or internal fluctuations in the network), induce transitions between two stable states. The network has two important features: (1) bistability; the network possesses two states (on and off), which stay stable in the absence of external input, allowing maintenance of representations by PFC neurons during 


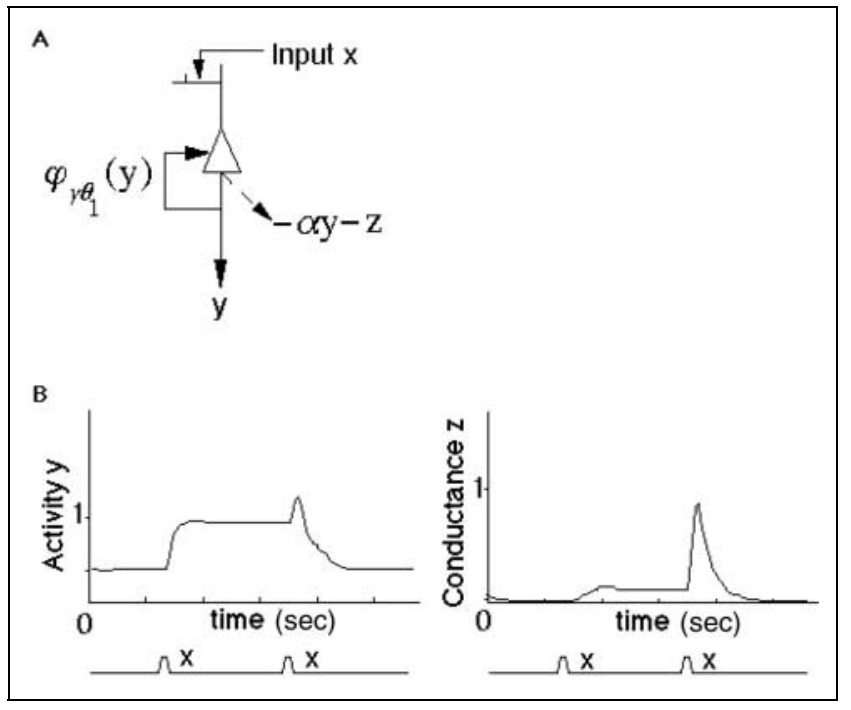

Figure 1. (A) Structure of the PFC network. Deep-layer pyramidal PFC neurons receive inputs from long-range cortical areas on superior layers and recurrent excitatory inputs from neighboring cortical columns on their basal dendrites. (B) Left: The activity $y(t)$ of the network can be switched on and off by transient excitatory inputs $x$ arriving on superficial layers. These inputs represent go-signals or noise (distracting stimuli/internal fluctuations). The property of bistability allows maintenance of representations during a delay period. Right: PFC pyramidal neurons possess intrinsic properties, as the slowly inactivating potassium conductance $z(t)$. This conductance is recruited at an intermediary level during the sustained activity of the neuron and increases rapidly with frequency $y$ when a new excitatory input arrives during the discharge, which can induce an intrinsic inhibition, furnishing a mechanism to stop the discharge. This mechanism allows both transitions on $\rightarrow$ off and off $\rightarrow$ on by the same excitatory input $x$.

the delay period; (2) the capacity to rapidly change between stable states with a transitory excitatory input. These two conflicting properties are important for updating representations within the PFC (Miller \& Cohen, 2001).

Dopaminergic neurons originating in the ventral tegmental area (VTA) and projecting to the PFC modulate working memory processes. Deficits of the delayed alternation task have been observed after pharmacological lesion of VTA neurons or of dopamine (DA) terminals in the PFC of monkeys (Brozoski, Brown, Rosvold, \& Goldman, 1979) and rats (Bubser \& Schmidt, 1990; Simon, 1981). Recent studies have revealed that DA D1 receptors are essential to working memory function in both the human and nonhuman primate PFC (Muller, von Cramon, \& Pollmann, 1998; Murphy, Arnsten, Goldman-Rakic, \& Roth, 1996; Williams \& GoldmanRakic, 1995; Sawaguchi \& Goldman-Rakic, 1991, 1994). Intra-PFC infusion of D1 receptors agonists (SKF 81297) or antagonists (SCH 23390) impaired performance of the delayed alternation task (Arnsten, 1997; Zahrt, Taylor, Mathew, \& Arnsten, 1997; Murphy et al., 1996). These D1 agonists/antagonists exert a long-lasting influence (several minutes or hours) on PFC pyramidal neurons (Gao, Krimer, \& Goldman-Rakic, 2001; Gulledge \& Jaffe,
2001; Seamans, Gorelova, Durstewitz, \& Yang, 2001; Zahrt et al., 1997).

In contrast to the long-lasting effect of D1 agonist or antagonist administration, DA is released at specific times of behavior (Schultz, 1997; Ljungberg, Apicella, \& Schultz, 1991), which is of particular importance to precisely modulate neuronal properties at the time scale of a cognitive task. Although the precise duration of the PFC postsynaptic effect of phasic DA release during performance of a cognitive task is still unclear, indirect evidence suggests that it could last a few seconds, as measured by electrophysiology, in vivo dialysis and voltammetry (Hasegawa, Blitz, Geller, \& Goldberg, 2000; Lewis \& O'Donnell, 2000; Gonon, 1997; see Methods).

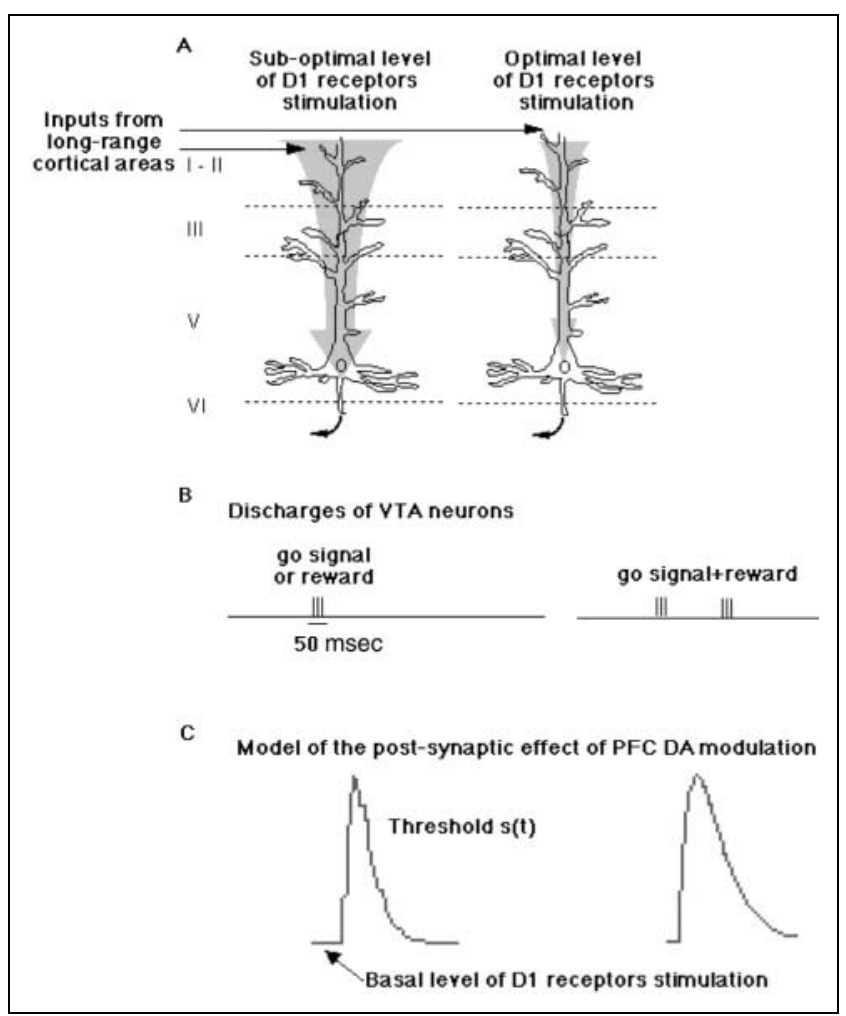

Figure 2. Model of the postsynaptic effect of dopamine in the PFC. The postsynaptic effect of phasic dopamine release in the PFC is determined by three factors: the direction of dopamine influence, the timing of dopamine release, and the duration of its postsynaptic action. (A) DA restricts inputs from long-range cortical areas targeting preferentially superficial layers of PFC pyramidal neurons (Yang \& Seamans, 1996). (B) In the delayed alternation task, VTA neuron discharges occur for go-signal or for reward (delivered for a correct alternation) (Ljungberg et al., 1991). (C) The postsynaptic effect of phasic dopamine release is modeled by a temporary threshold $s(t)$ restricting inputs arriving on apical dendrites of deep-layer pyramidal neurons. The duration of the postsynaptic effect of DA could last a few seconds (Lewis \& O'Donnel, 2000; Gonon, 1997). Two different durations of this threshold are considered to take into account the precise time of VTA firing during the delayed alternation task. Left: Following an incorrect trial (go-signal alone), the duration of this threshold is supposed to last a few seconds. Right: Following a correct trial, the threshold reflects the sum of the duration of the postsynaptic effect of DA delivered for the go-signal and the reward. 
The relation between the cellular effect of PFC D1 DA receptors stimulation and the level of working memory performance remains poorly understood. Previous models of DA modulation in the PFC have been situated at different levels of abstraction, either using connectionist networks to link reduced DA PFC turnover to performance in various cognitive tasks (Braver, Barch, \& Cohen, 1999; Cohen \& Servan-Schreiber, 1992; Cohen, Braver, \& O'Reilly, 1996; Servan-Schreiber, Printz, \& Cohen, 1990) or biophysically detailed models of spiking neurons, linking the firing rate of PFC neurons to the change of their biophysical properties induced by variations of DA receptors stimulation (Durstewitz, Kelc, \& Gunturkun, 1999, Durstewitz, Seamans, \& Sejnowski, 2000).

Here we chose an intermediary level of modeling that allows association between the cellular effect of PFC D1 receptors stimulation and performance in the delayed alternation task. The originality of our model is to combine the long-lasting effect of D1 agonists/antagonists administration and the short-term postsynaptic

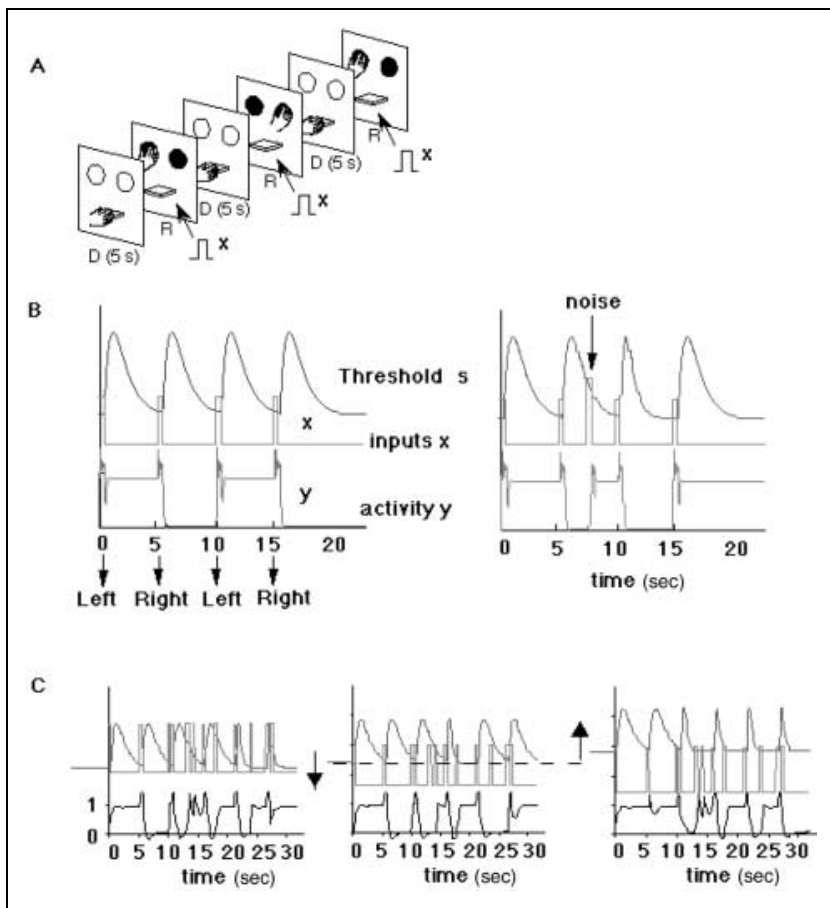

Figure 3. (A) Structure of the delayed alternation task. Successive go-signals $x$ (simultaneous lightning of the two circles) required to alternate between two responses (right and left) separated by a delay of 5 sec. (B) Left: Time course of the network activity $y(t)$ and time course of the postsynaptic effect of dopamine (temporary threshold) in the PFC for successive transitory excitatory inputs $x$. Right: When noise is added to the system, unexpected transitions can occur before the next go-signal if the noise is higher than the temporary threshold. (C) Examples of activity $y(t)$ of the network when noise is added to the system and the basal level of dopamine D1 receptors stimulation is decreased (left) or increased (right) as compared to an arbitrary baseline (middle). The arrows pointing to the bottom/top indicate a decrease/increase of the basal level of D1 receptors stimulation (injection of D1 antagonists/agonists).

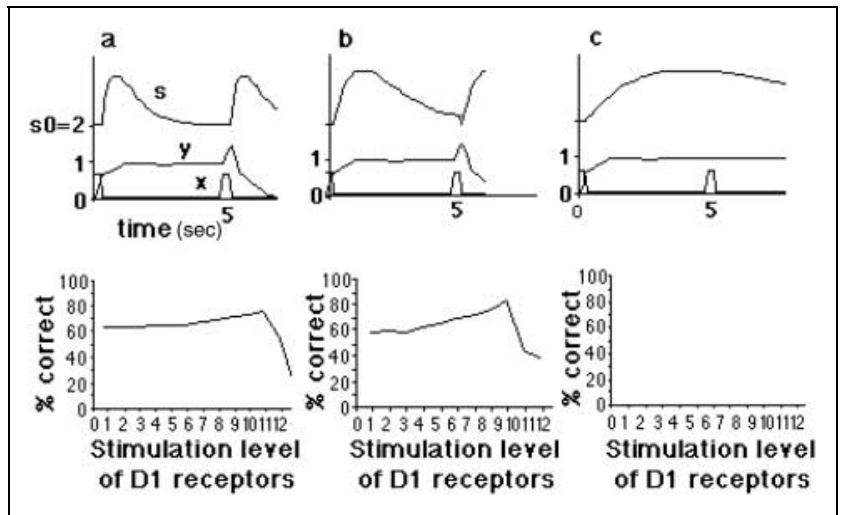

Figure 4. Top: Time course of the network activity $y$ and of the postsynaptic effect of dopamine (temporary threshold $s$ ) in the PFC for two successive transitory excitatory inputs $x$ when the time constant of the threshold is lower (case a), adapted (case b) or higher (case c) than the delay. Bottom: Network performance for increasing level of D1 receptors stimulation in cases $\mathrm{a}, \mathrm{b}$, and c. Noise is distributed according to a Poisson process of mean 5 sec.

effect of phasic DA release (Figure 2, see Methods), and to show how they relate to behavioral performance during the delayed alternation task. The postsynaptic effect of phasic DA release is modeled as a temporary threshold restricting the inputs arriving on apical dendrites of deep-layer pyramidal neurons (Figure 2C, see Methods). The duration of the postsynaptic effect of phasic DA release in the PFC is important to precisely regulate whether incoming inputs arriving on the PFC network switch or not its stable states. As the exact duration of this restriction is currently uncertain, the model evaluates the behavioral performance when this duration is or is not adapted to the delay of the task (Figure 4, top row). In addition, injection of D1 receptors agonists (antagonists) is modeled as an increase (decrease) of the basal level of dopaminergic D1 receptors stimulation (Figure 3C). The performance of the network is tested during progressive increase of the basal level of DA D1 receptors stimulation when noise is added to the inputs (Figures 3B and 4).

\section{RESULTS}

Figure 4a (bottom) shows the level of the network performance when the time constant of the threshold is lower than the delay. Performance follows an inverted U-curve when D1 receptors stimulation increases. The optimal level of performance is lower and obtained for higher level of D1 receptors stimulation compared to when the time constant of the threshold is adapted to the delay (see Figure $4 \mathrm{~b}$, bottom). Indeed, a low time constant of the threshold reduces the duration of restriction of incoming inputs, making it more likely for noise to disrupt memory for a given level of D1 receptors stimulation. Importantly, performance falls abruptly below 50\% correct after the optimal performance is reached, because the temporary 


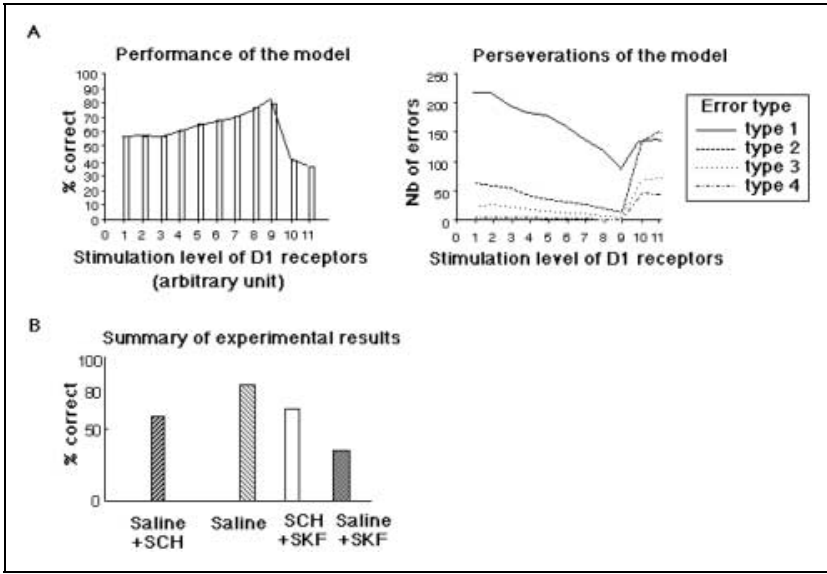

Figure 5. (A) Left: Results of the simulated network performance for increasing level of D1 receptors stimulation when the time constant of the threshold is adapted to the duration of the delay. Right: Quantification of error types for $n$ successive delays as a function of the level of D1 stimulation $(n=1500)$. Perseverations are quantified by counting two successive errors (e.g., on-on-on-off is a succession of two errors). A perseveration of type 1 is thus the repetition of length 1 of the same activity (e.g., on-on). Noise is distributed according to a Poisson process of mean $5 \mathrm{sec}$. (B) Summary of experimental performance in the delayed alternation task for increasing level of PFC D1 receptors stimulation. Saline + SCH: systemic injection of saline and PFC injection of D1 receptors antagonist SCH $23390(0.035 \mathrm{mg} / \mathrm{kg})$; SCH + SKF: systemic injection of SCH $23390(0.03 \mathrm{mg} / \mathrm{kg})$ and PFC injection of D1 receptors agonist SKF $81297(0.1 \mu \mathrm{g})$; Saline + SKF: systemic administration of saline and PFC injection of SKF 81297 (0.1 $\mu \mathrm{g})$. (Adapted from Zahrt et al., 1997; Murphy et al., 1996).

threshold no longer restrict go-signals for high levels of receptors stimulation.

Figure 4b (bottom) represents the behavior of the network as a function of the level of D1 receptors stimulation when the time constant of the postsynaptic effect of DA is adapted to the delay. The performance changes with the level of D1 receptors stimulation according to an asymmetric U-curve. D1 antagonists induce a random behavior ( $\sim 50 \%$ of success), because noise easily induces transitions between go-signals. For high levels of D1 receptors stimulation, performance drops below chance level, reflecting that the network remains in the same state for successive trials (perseverations), because the go-signals are restricted by the postsynaptic effect of DA. However, performance does not continue to drop abruptly for further increase in D1 receptors stimulation (as was the case when the time constant of the threshold was lower than the delay). Indeed, when an error occurs, the absence of reward delivery reduces the duration of the temporary restriction of inputs, thereby allowing incoming noise and/or go-signals to switch between states (see Figures 2C and 3C, middle).

Finally, Figure 4c (bottom) shows that if the time constant of the threshold is very large as compared to the delay, all the inputs (go-signals and noise) are restricted. No transition between states is therefore possible, leading to a null level of performance.

\section{Perseverations}

In order to analyze the performance of the network more precisely, we also quantified perseverations. As the network performance only corresponds to experimental data (see Discussion) when the time constant of the threshold is adjusted to the delay, we only quantified perseverations in this case (Figure 5A, right). At low levels of D1 stimulation, the number of perseverations is high and then decreases with an increase of the level of D1 stimulation, until reaching a minimal level, which corresponds to the optimal level of performance. When D1 receptors stimulation increases further (for injection of D1 agonists), the perseverations strongly increase. It is important to note the different nature of perseverations appearing for D1 antagonists and for D1 agonists. Perseverations present for D1 antagonists are due to the fact that the delayed alternation task only comprises two possible choices. Thus, as the noise easily induces transitions for low levels of D1 receptors stimulation, there is a high probability to make an inopportune transition between two go-signals. This explains why a large amount of Type 1 perseverations (repetition) are observed for D1 antagonists. If the choices were distributed not among two responses (right and left) but among $n$ possible responses $(n>2)$, there would be only $1 / n$ chances to make the same choice at each trial. Therefore, perseverations appearing for D1 antagonists are not the mark of a real behavior of perseveration but come from the inherent structure of the task. In contrast, the high number of true perseverations of the network (errors of type $>1$ ) observed for D1 agonists are due to the fact that both distractors and go-signals cannot switch the current state. Long perseverations are thus observed, leading to performance correct below 50\%.

\section{Effect of the Delay Period Duration on Performance}

We also examined how distinct delay periods affect the performance of the network. Three delay durations

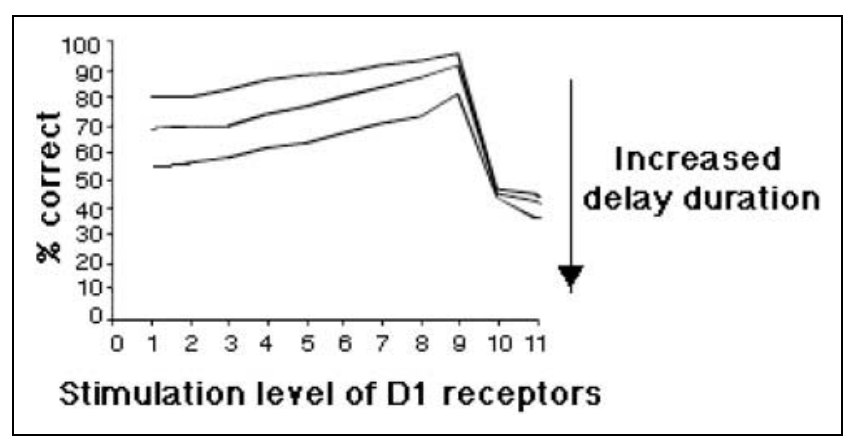

Figure 6. Percent correct of performance of the model for short ( $2 \mathrm{sec}$, top curve), standard ( $5 \mathrm{sec}$ ) and long ( $8 \mathrm{sec}$, bottom curve) delay periods. Noise is distributed according to a Poisson process of mean $5 \mathrm{sec}$. 
$(2,5,8 \mathrm{sec})$ were tested, which reflect the delay periods used in three groups of animals, each trained with a particular delay. In each group, the duration of the postsynaptic effect of DA is supposed to be adapted to a particular delay. Comparison between the different delay durations shows that increasing the delay decreases the model's performance at all levels of DA D1 receptors stimulation (Figure 6). This is illustrated by a translation of the performance curve when the delay increases. The reduction of performance is explained by the fact that the probability that noise induces transitions increases with the duration of the delay.

\section{DISCUSSION}

Our model allows a better understanding of the relation between the cellular action of DA on a simple PFC neural network and the level of performance of the delayed alternation task. It conciliates the short-term postsynaptic effect of phasic DA release and the long time scale variation of the basal level of DA receptors stimulation occurring after D1 agonists and antagonists injection. The model explains how phasic DA finely regulates inputs on prefrontal network and how the inverted U-curve of performance is obtained with an increase in the level of D1 receptors stimulation.

Experiments in rats showed that systemic or local PFC injection of the D1 receptors antagonist, SCH 23390, induce deficits in performance of the delayed alternation task (Zahrt et al., 1997; Murphy et al., 1996). Furthermore, local injection of the D1 receptors agonist, SKF 81297, leads to a reduction in the performance of this task (Zahrt et al., 1997). The deficits induced by SKF 81297 are inverted by pretreatment with SCH 23390, indicating that an optimal level of D1 receptors stimulation is crucial for correct performance of this task (Figure 5B). This inverted U-curve explains previous contradictory results. For instance, a paradoxical improvement of performance is observed for very low doses of D1 agonist (0.0001 mg/kg) in aged monkeys who have a natural reduction of extracellular DA in the PFC (Cai \& Arnsten, 1997; Arnsten, Cai, Murphy, \& Goldman-Rakic, 1994). This improvement may be explained by considering the basal level before injection of D1 agonist. If injection of D1 agonist is done for a suboptimal basal level of DA D1 receptors stimulation, as is the case for aged monkeys, a small dose of DA D1 agonist will improve performance. Inversely, D1 antagonists can improve performance when the basal level of D1 receptors stimulation is higher than the optimal level of stimulation. For instance, stress increases the level of stimulation of dopaminergic receptors in rats or monkeys (Arnsten \& Goldman-Rakic, 1998; Deutch, Clark, \& Roth, 1990), but deficits induced by stressful tasks in spatial working memory are blocked by the D1 receptors antagonist SCH 23390 (Arnsten, 1997; Murphy et al., 1996, Murphy, Roth, \& Arnsten, 1997).
Comparison of the network performance with experimental results obtained in rats or monkeys indicates that the simulated curve of performance is not symmetric and that performance abruptly decreases after optimal level of DA receptors stimulation. The asymmetry of the inverted U-curve may be attributed to the fact that an identical dose of D1 agonists or antagonists does not have the same behavioral effect. In fact, the relation between the dose injected and the level of DA stimulation is probably logarithmic (Martinot et al., 1995). The results of our model thus show a qualitative nonmonotonic relationship between the behavioral performance and the level of D1 stimulation. For high levels of D1 receptors stimulation, which correspond to D1 agonist injection, both go-signals and distracting stimuli were restricted, causing the network to remain in the same state during successive trials. This prediction of increased perseverations with an increase in the level of D1 stimulation is confirmed by local injection of D1 agonists in the PFC of rats (Zahrt et al., 1997). Our model also predicts that local PFC injection of D1 antagonists would lead to a chance level of performance. This prediction is confirmed experimentally as the percentage of correct responses is close to $50 \%$ in rats after medial PFC injection of D1 antagonists (Zahrt et al., 1997). Finally, the prediction that an increase in the delay period would decrease network performance remains to be tested experimentally (Figure 6).

\section{Precise Regulation of Postsynaptic Effect of DA Modulation}

One important prediction of the model is that the postsynaptic duration of DA may be adjusted to the delay period of the task. This may be possible if DA released after phasic VTA discharge is adapted to the delay duration, implying that the frequency of VTA neurons discharge depends upon the delay of the task (discharging more for long delays). This prediction is supported by the observation that DA released in the striatum after substantia nigra stimulation is both frequency- and current-dependent (Garris, Christensen, Rebec, \& Wightman, 1997). Furthermore, Richardson and Gratton (1998) have shown by voltammetry in the rat that under delayed reinforcement conditions, lever presses were followed by DA PFC signal increases that were time-locked to the delay duration (Richardson \& Gratton, 1998). Adaptation of the postsynaptic duration of DA to the delay is made possible by the close relationship between sustained activities and the time of VTA discharges. VTA neurons code the error of reward prediction (Schultz, 1997, Montague, Dayan, \& Sejnowski, 1996), and this signal, furnished to the PFC (Taber, Das, \& Fibiger, 1995), is itself elaborated on the basis of reward prediction delivered by the PFC (Watanabe, 1996). Thus, PFC neurons, which show enhanced activity with increase desirability of an expected reward 
(Leon \& Shadlen, 1999; Tremblay \& Schultz, 1999; Watanabe, 1996), could dynamically regulate their own DA level, as supported by the increase of PFC DA extracellular level observed after performance of the delayed alternation task (Watanabe, Kodama, \& Hikosaka, 1997).

Current theories consider phasic DA as a teaching signal for reinforcement learning (Suri \& Schultz, 2001; Contreras-Vidal \& Schultz, 1999; Montague et al., 1996; Friston, Tononi, Reeke, Sporns, \& Edelman, 1994). DA responses transfer during learning from primary rewards to reward-predicting stimuli. This role of DA in learning is compatible with our proposed threshold function of phasic DA, which concerns a fundamental role of modulatory information processing. Phasic DA could, at the same time than protecting sustained activities from noise, form connections during novel situations. This learning role of DA, which is not accounted by our model, might be of primary importance to adapt the postsynaptic effect of DA after unexpected change of the delay duration. Such rapid delay variation should be associated to an increase of errors and should lead to an adaptation of DA discharge frequency after learning.

\section{Comparison to Previous Models of PFC DA Modulation}

In our model, the basal level of DA receptors stimulation (protecting delay activity against interfering stimulus) has a similar functional role like the one proposed by previous DA PFC models (Durstewitz et al., 1999; Cohen \& Servan-Schreiber, 1992). For instance, Durstewitz et al. (1999, 2000) proposed that DA enables PFC network to hold active neural representations of goalrelated information and thereby to protect goal-related delay activity against interfering stimulus. They designed a detailed model showing that DA influence on biophysical properties of PFC neurons is appropriate to fulfill this function. Using a more abstract class of network (Parallel Distributed Processing models), Cohen and Servan-Schreiber (1992), Cohen et al. (1996), and Servan-Schreiber et al. (1990) have proposed to link DA function to the performance of different cognitive tasks. For these authors, DA increases signal-to-noise ratio (gain parameter) of the sigmoid function of formal neurons, which would facilitate both excitatory and inhibitory inputs. Such facilitation is unlikely to occur in light of recent electrophysiological studies (see Methods).

The DA PFC models mentioned above have postulated that the effect of DA is time-independent, an assumption that can no longer be supported (Schultz, 1997; Schultz \& Dickinson, 2000). The hypothesis that DA increases signal-to-noise ratio in a time-independent manner is insufficient to explain the U-curve of performance obtained in the delayed alternation task. According to this hypothesis, performance would increase monotonically with an increase of DA receptors stimulation until reaching an optimal level of performance, and would then fall abruptly to a null level of performance because of restriction of all go-signals. In our model, it is the combination of the basal level of D1 receptors stimulation, which increases the signal-to-noise ratio, and of the postsynaptic effect of phasic DA release, which more specifically regulates activities at the time scale of the task, that explains the inverted U-curve of performance.

Recent theories of PFC DA modulation have introduced a gating mechanism reflecting phasic DA release at the precise time of presentation of the cue and probe stimuli (Braver et al., 1999). However, experimental studies suggest that the postsynaptic effect of this phasic DA release occurs after presentation of the stimuli (Lewis \& O'Donnell, 2000; Gonon, 1997). Furthermore, DA may not be able to gate PFC inputs at the precise time of their arrival because the time necessary for a stimulus to induce VTA discharge (100-150 msec, Ljungberg et al., 1991), added to the latency necessary for DA to act at postsynaptic sites (200 msec from VTA to the striatum; Gonon, 1997; Hille, 1992), is longer than the time necessary for a stimulus to reach PFC (around $100 \mathrm{msec}$ ) (Thorpe, Fize, \& Marlot, 1996). Rather than gating stimuli at the time of their arrival, our model proposes that phasic DA release restricts incoming stimuli for a period of time after VTA discharges. Importantly, the gating theory of DA at the time of stimuli presentation and our own theory make different predictions in regard to the role of errors in learning. In our view, the absence of DA delivery following an error allows the updating of PFC representation (because the restriction of inputs is reduced), which may be useful to learn the correct behavior to be adopted. This is in accordance with the current view that behavioral learning depends on the coding of reward prediction error by DA neurons (Waelti, Dickinson, \& Schultz, 2001; Schultz \& Dickinson, 2000). In contrast, according to the gating theory, updating of representations is not done after each error but after phasic DA signal for reward or reward predicting stimuli (Braver et al., 1999). These distinct predictions will be useful to test the validity of these two models in future learning paradigms.

Our model shows that supposing that the overall role of DA is to reduce inputs to pyramidal neurons in a phasic and tonic fashion is sufficient to explain the inverted U-curve of performance and the nature of errors (distraction, perseveration) observed in the delayed alternation task. More complex models are certainly possible. For instance, the balance between the roles of inhibitory interneurons and pyramidal cells may depend upon the level D1 receptors stimulation (Goldman-Rakic, Muly, \& Williams, 2000) and/or the neuromodulatory effects of D1 receptors activation may be state-dependent (Maurice, Tkatch, Meisler, Sprunger, \& Surmeier, 2001; Wang \& O’Donnell, 
2001). Future computational simulations will need to test such hypotheses and to integrate the phasic/tonic mode of DA modulation.

In conclusion, we propose a new theory of DA PFC modulation that challenges previous connectionist models. Based on current physiological and electrophysiological properties of PFC and DA neurons, we offer a biologically plausible neural network that links behavioral performance during a working memory task and DA PFC modulation. The model allows us to understand, with a prefrontal network that can maintain and stop sustained activities after transitory excitatory inputs, how the level of DA D1 receptors stimulation can lead to the inverted U-curve of performance obtained in the delayed alternation task. The model led us to interpret differently the reduction of performance for D1 antagonists and D1 agonists, which could a priori seem similar when only considering the performance curve. The model shows that perseverations obtained for D1 agonists come from a defect to take pertinent stimuli into account, and that distractability is obtained for D1 antagonists. These results may be highly relevant to understand cognitive deficits in schizophrenia, which have been associated with a reduction of PFC D1 receptors stimulation (Okubo et al., 1997). The fundamental contribution of the model is to combine a functional role of phasic DA release and of the basal level of DA receptors stimulation in the PFC and to link the level of performance to the cellular action of DA in a neural network. Finally, the model generates the testable prediction that the duration of the postsynaptic action of DA is adjusted to the length of the delay. We look forward for further testing of our DA PFC hypothesis in other cognitive tasks than the delayed alternation task.

\section{METHODS}

\section{Physiological Basis of the Model}

\section{Architecture of the Network}

Pyramidal cells constitute the major portion of neurons with sustained activities and motor responses (Silva, Amitai, \& Connors, 1991; Sawaguchi, Matsumura, \& Kubota, 1990; Connors, Gutnick, \& Prince, 1982; Fuster, 1973), whereas neurons in superficial layers are linked to the reception of sensory information (Sawaguchi et al., 1990). PFC connectivity on deep-layer pyramidal neurons is organized in two principal information flows: (1) external inputs from long-distance areas arrive on superior-layer (II-III) neurons and are integrated by the apical tree of these pyramidal neurons (Kuroda, Murakami, Kishi, \& Price, 1995; Jones, 1984); (2) inputs from neighboring cortical columns arrive on the deep layer and are integrated on basal dendrites and soma (Kritzer \& Goldman-Rakic, 1995; Levitt, Lewis, Yoshioka, \& Lund, 1993). In turn, these pyramidal neurons send glutamatergic efferents to the nucleus accumbens and to the VTA (Taber et al., 1995). The network architecture represents a local circuit of deep-layer PFC pyramidal cells connected in local excitatory recurrent networks via their basal dendrites, and receiving external inputs via their apical dendrites (Figure 1A).

\section{Model of Sustained Activity}

Sustained activity is found in numerous cortical and subcortical areas, suggesting the existence of general mechanisms for their generation. Both PFC and entorhinal cortex neurons have the particular ability to maintain sustained activity when distracting objects are presented during the delay (Suzuki, Miller, \& Desimone, 1997; Miller, Erickson, \& Desimone, 1996b). By contrast, neither temporal cortex neurons (Miller et al., 1996b), nor posterior parietal cortex neurons (Constantinidis \& Steinmetz, 1996; di Pellegrino \& Wise, 1993) survive the presentation of intervening stimuli. The role of sustained activity in memory tasks is demonstrated by two observations: The percentage of PFC neurons which exhibit such activity is proportional to the correct performance of the animal (Alexander, 1982; Fuster, 1973), and the disruption of sustained activity by distracting stimuli often reflects an error made by the monkey (Miller et al., 1996a, 1996b). The origins of sustained activities of pyramidal neurons have either been attributed to recurrent connections, consistent with the fact that they can be found at all stages of cortico-thalamocortical loops (Goldman-Rakic, 1995; Levitt et al., 1993), and/or to intrinsic neuronal mechanisms (ionic channels) (Camperi \& Wang, 1998; Delord et al., 1997; Marder et al., 1996).

We model spatially selective delay activity by the mean discharge frequency of a population of neurons coding anticipation of a right response by a high frequency of discharge (on state) and the anticipation of a left response by a low frequency of discharge (off state). The opposite pattern of discharge would be embodied in a second population of neurons and will not be modeled here. We designed the following network to model these sustained activities:

$$
\begin{gathered}
\tau_{y} \frac{\mathrm{d} y}{\mathrm{~d} t}=-\alpha y+\varphi_{\gamma \theta_{1}}(y)+I_{x, s}-z \\
\tau_{z} \frac{\mathrm{d} z}{\mathrm{~d} t}=-\beta z+\varphi_{\Gamma \theta_{2}}(y)
\end{gathered}
$$

where $\varphi_{a b}(u)=1 /(1+\exp (-a(u-b)))$. In this system of differential equations, $\theta_{1}$ represents the threshold on basal dendrites, $s$ is the threshold on apical dendrites (see implementation of DA modulation), $\alpha$ and $\beta$ are constants of passive decay, $\tau_{y}$ and $\tau_{z}$ are arbitrary time constants and $\gamma$ and $\Gamma$ correspond to the gain of sigmoid functions. 
This network is able to switch on and off sustained activities after a transitory excitatory input $x$. Equation 1 describes the dynamic of the mean activity $y$ of the network presented in Figure 1A. The variable $y(t)$ is equivalent to a frequency or to a probability of discharge of the neuronal population (Figure 1B, left). Equation 2 describes the dynamic of a slow variable that can either be viewed as an intrinsic property of the neurons, such as the slowly inactivating potassium current (Delord, Klaassen, Burnod, \& Guigon, 1996, 1997), or as feedback inhibition from inhibitory interneuron. Indeed, previous models have shown that transitory excitatory inputs may induce both transitions off $\rightarrow$ on and on $\rightarrow$ off when neurons possess a slowly inactivating potassium conductance, which is the case for pyramidal neurons of the rat frontal cortex (Delord et al., 1997; Hammond \& Crépel, 1992). This mechanism is important because it allows the same excitatory input to induce both on $\rightarrow$ off and off $\rightarrow$ on transitions.

In Equation 1, the first term $-\alpha y$ describes the passive decay of activity and insures stability of both states. The second term $\varphi_{\gamma \theta_{1}}(y)$ represents recurrent inputs on basal dendrites and insures sustained activities representing memory of the excitatory input that induced it. The term $I_{x, s}$, a nonzero constant if $x>s$ and 0 otherwise, represents the signal induced by an excitatory transitory input $x$ arriving on apical dendrites, which is restricted by the threshold $s$, the postsynaptic action of DA (described in Implementation of DA modulation). This term $I_{x, s}$ can induce transitions between stable states (on $\rightarrow$ off or off $\rightarrow$ on). Finally, the term $z$ is a variable activated by the discharge and inhibiting it. It allows stopping the regenerative discharge by the transitory input $x$ (Figure 1B, right). The first term of Equation 2 describes the passive decay of $z$, which allows its convergence, while the second term (sigmoidal) describes the increase of $z$ with the increase of $y$ activity. Figure 7 displays the phase plane analysis of this network.

\section{Model of DA Modulation in the PFC}

Deep-layer PFC pyramidal cells are densely innervated by dopaminergic fibers (Joyce, 1993; Berger, Gaspar, \& Verney, 1991). They are the major neuronal population expressing specific D1 receptor-related proteins (Smiley, Levey, Ciliax, \& Goldman-Rakic, 1994). As long-range cortical inputs arriving on deep-layer PFC pyramidal neurons target their apical dendrites, the model primarily considers DA influence at this site because longrange cortical inputs transmitting external signals have a major role in switching between stable states. The reason for the specific role of $\mathrm{D} 1$, but not $\mathrm{D} 2$ receptors, in modulating working memory may be related to the fact that D1 receptors are at least 20-fold more abundant in the PFC than D2 receptors (Lidow, Goldman-Rakic, Gallager, \& Rakic, 1991). Recent studies have proposed that D1 receptors stimulate acts via modulation of

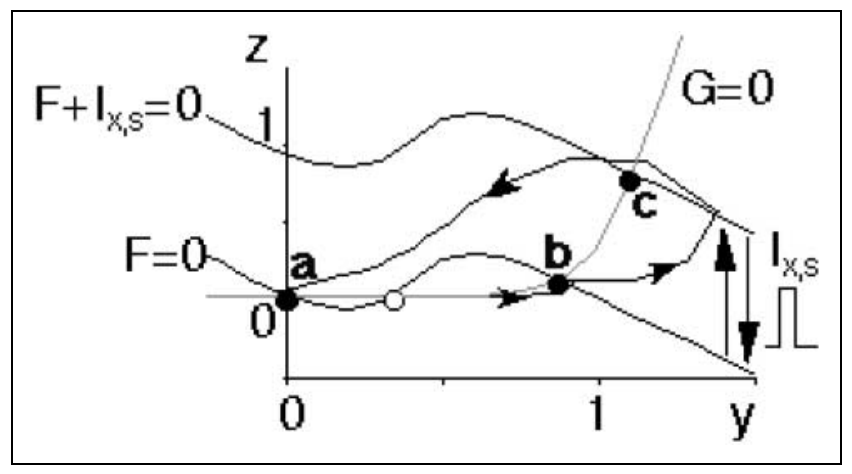

Figure 7. Trajectory of the phase point $(y(t), z(t))$ in the phase plane for two successive transient excitatory inputs $I_{x, s}$, respectively, inducing transitions from a (off) to $\mathrm{b}$ (on) and from $\mathrm{b}$ to $\mathrm{a}$ (on $\rightarrow$ off). The $y$-nullcline corresponds to $F+I_{x, s}=0$ and the $z$-nullcline corresponds to $G=0$. The transient input $I_{x, s}$ briefly translates the curve $F=0$, creating a temporary stable state c. However, for an appropriate duration $I_{x, s}$, the system does not converge to c, but comes back to the stable state a. The position of c (intersection of $F+I_{x, s}=0$ and $G=0$ ) explains that the second transitory input $x$ briefly increases both $y$ and $z$ (see Figure $1 \mathrm{~B}) . a \cong(0.0223,0) ; b \cong(0.8871,0.1046) ; \alpha=1, \beta=0.5$; $\tau_{y}=2, \tau_{z}=1, \gamma=10, \Gamma=10, \theta_{1}=0.4, \theta_{2}=1.2$.

glutamate release at synaptic triads of pyramidal neuron dendritic spines (Gao et al., 2001; Seamans, Durstewitz, Christie, Stevens, \& Sejnowski, 2001).

The effect of DA modulation is determined by the direction of its action, the precise time of phasic DA release, and the duration of its postsynaptic action:

Direction of DA action. In vivo data suggest that DA has a general inhibitory effect on PFC activity, with one of its primary effects being the modulation of excitatory inputs on pyramidal neurons (Jedema \& Moghddam, 1996; Williams \& Goldman-Rakic, 1995; Cepeda, Radisavljevic, Peacock, Levine, \& Buchwald, 1992). VTA stimulation decreases PFC neuron firing (Lewis \& O’Donnell, 2000; Jay, Glowinski, \& Thierry, 1995; Ferron, Thierry, Le Douarin, \& Glowinski, 1984). This action is likely to occur from indirect action on GABAergic neurons projecting to pyramidal cells (Seamans, Gorelova, et al., 2001; Pirot et al., 1992; Godbout, Mantz, Pirot, Glowinski, \& Thierry, 1991; Ferron et al., 1984). However, the electrophysiological effect of PFC DA modulation is still controversial, with data either supporting an increase or a decrease of discharge frequency of sustained activities during delay (Williams \& GoldmanRakic, 1995; Sawaguchi et al., 1990; Sawaguchi \& Goldman-Rakic, 1994). For this reason, our PFC DA model did not consider the DA influence on discharge frequency. Recent simulations of the DA influence of intrinsic ionic and synaptic currents have led to a more coherent functional role of DA in the context of working memory performance (Durstewitz et al., 1999, 2000). DA has been proposed to increase the robustness of representations encoding goal-related information via D1 receptors modulation. This overall effect of DA on the deep-layer PFC pyramidal neurons is in accordance with 
the fact that, in vitro, DA restricts inputs arriving on apical dendrites by increasing the electronic distance between the distal and the proximal dendritic regions (Gorelova \& Yang, 1997; Zahrt et al., 1997; Yang \& Seamans, 1996) (Figure 2A).

Time of DA release. After learning the delayed alternation task, VTA neurons discharge both after triggers and after rewards (Ljungberg et al., 1991). However, when an error occurs, reward is not delivered, and DA neurons do not fire (Ljungberg et al., 1991) (Figure 2B, left). It has to be noted that contrary to other delayed response tasks, DA released after reward delivery persists after overtraining, which has been attributed to the fact that reward serves both as task reinforcer and as important stimulus for the continuous adaptation to the target in the next trial during the delayed alternation task (Ljungberg et al., 1991).

Time course of the postsynaptic effect of DA. Two important functional points remain to be considered: (1) the time when DA, released in the PFC after VTA firing, starts to restrict inputs arriving on PFC pyramidal neurons and (2) the duration of DA postsynaptic action.

(1) During the delayed alternation task, stimuli induce VTA and PFC firing after a latency of 100-200 msec (Ljungberg et al., 1991). In addition, a latency (around $200 \mathrm{msec}$ ) is likely to exist between VTA firing and DA action on PFC pyramidal neurons, because spike responses of certain striatal neurons population receiving excitatory inputs are delayed by $200 \mathrm{msec}$ following DA fibers stimulation (Gonon, 1997). This latency corresponds to the slow conduction velocity of the dopaminergic axons and to the time required to activate adenylate cyclase through G-protein-mediated messenger systems (Gonon, 1997; Hille, 1992). Thus, during the delayed alternation task, DA released in the PFC after a go-signal should start to restrict inputs arriving on superficial layers of pyramidal neurons a few hundred milliseconds after this signal.

(2) The exact duration of postsynaptic DA effect in the PFC during a cognitive task is still unknown. An in vivo study of the DA transmission kinetic mediated by D1 receptors suggests that it could last a few seconds. Combined electrical stimulation of the rat medial forebrain bundle mimicking the spontaneous activity of dopaminergic neurons and real time electrochemical monitoring of the resulting DA overflow in the extracellular space has shown that the postsynaptic effect of DA on one type of striatum discharge is to increase their frequency up to $1 \mathrm{sec}$ (Gonon, 1997). As the halflife for clearance is approximately $2 \mathrm{sec}$ in the rat medial PFC, while in the striatum it is only $0.06 \mathrm{sec}$ (Gonon, 1997; Garris, Ciolkowski, Pastore, \& Wightman, 1994), it is likely that DA PFC postsynaptic effect can last a few seconds. This assumption is more directly supported by the observation that PFC pyramidal neurons, which exhibit in vivo a bistable membrane potential, can be switched to the up state for several seconds following electrical stimulation of the VTA mimicking burst firing (Lewis \& O'Donnell, 2000). Furthermore, we hypothesized that the postsynaptic effect of D1 receptors stimulation returns to baseline following clearance of DA from the extracellular space. More complex models may include a drift of the baseline level of the temporary restriction of inputs by phasic DA release during performance of the task. A possible functional role of this drift may be to restrict inputs arriving on PFC network more importantly at the end of the task.

\section{Implementation of DA Modulation}

We assume that the effect of phasic DA release is to restrict, for a few seconds, excitatory inputs arriving on superficial layers, thereby protecting apical dendrites of deep-layer PFC pyramidal neurons from incoming noise. As the exact duration of this restriction of inputs remains to be determined, the model evaluates the behavioral performance when this duration is or is not adapted to the delay of the task. The postsynaptic effect of phasic DA release is modeled as a threshold temporary restricting inputs arriving on apical dendrites of pyramidal neurons (Figure 2C). The duration of this threshold depends on the amount of DA released. The basal level of DA stimulation, which is increased (respectively decreased) by injection of DA D1 agonists and antagonists, represents the baseline of this threshold. Formally, the postsynaptic effect of DA is given by:

$$
s(t)=s_{0}+k_{i}\left(t-t_{i}\right)_{e}^{-\left(t-t_{i}\right) / \tau_{i}}, \quad t \geq t_{i}
$$

In Equation 3, $s_{0}$ corresponds to the basal level of DA receptors stimulation and the second term is the postsynaptic effect of phasic DA release (alpha function of amplitude $k_{i}$ ). It increases rapidly after each phasic DA release at time $t_{i}$ and decays progressively with a certain time constant $\tau_{i}$. The threshold $s(t)$ can vary in two ways, either by a modification of the basal level of dopaminergic receptors stimulation, or by a variation of phasic DA release. The basal level of DA D1 receptors stimulation is not modified by phasic DA release because the latter is rapidly recaptured (Parsons \& Justice, 1992).

\section{Model of the Delayed Alternation Task}

The monkey task is to depress an illuminated hold key for several seconds in order to darken it and to light two choice keys, then to press the correct key for that trial (e.g., the left) in order to obtain a reward. A single press on either choice key darkened both and relit the hold key, starting the next trial. A correct sequence is: hold center, press left (reward), hold center, press right (reward), and so forth (Figure 3A). The network described previously is used to model execution of the delayed alternation task, which is supposed to be 
established after learning. The task is simulated in the following way. External inputs $x$, with fixed amplitude, representing the go-signals (light of the two choice keys) are presented during $40 \mathrm{msec}$ all $5 \mathrm{sec}$ (delay interval). These go-signals induce a discharge of VTA neurons, whose terminals release DA, which in turn temporarily restricts inputs to the PFC network. In the optimal case, the go-signals exceed the $s$ threshold, which induce a motor response corresponding to the memorized state and a transition between stable states (Figure 3B, left).

\section{Addition of Noise to the Network}

The network presented previously performs the delayed alternation task without error (Figure 3B, left). In order to study the performance of the network in disrupted conditions when D1 agonists and antagonists are injected in the PFC, noise is added to the inputs (Figure 3B, right). This noise (duration of $40 \mathrm{msec}$ ) can be considered as internal fluctuations of the network or as being from external origin (distracting stimuli). It follows a Poisson process of fixed amplitude and of mean chosen in order to reach $80 \%$ of correct performance at the optimal level of D1 receptors stimulation, which corresponds to the level of performance achieved by rats after learning the task (Zahrt et al., 1997). This noise can induce transitions between two go-signals when exceeding the threshold $s(t)$. An error can thus occur, corresponding to an absence of alternation. In such a case, the reward is not delivered and VTA neurons do not discharge at the time of reward delivery (Ljungberg et al., 1991).

\section{Distinct Threshold Durations for Correct/Incorrect Trials}

Two durations of postsynaptic effect of DA are considered for incorrect and correct alternations. (1) When an error occurs, reward is not delivered. Thus, DA released in the PFC is only induced by the go-signal. In this case, the duration of postsynaptic action is modeled by a short threshold $s(t)$ on inputs (Figure 2C, left). (2) After a correct alternance, VTA neurons discharge both following reward and trigger (Ljungberg et al., 1991). In such successful trial, the postsynaptic effect of these two successive releases of DA, separated by 300-400 msec, can be modeled as one cumulative long threshold (Figure 2C, right). Two type sets of parameters $k_{i}$ and $\tau_{i}$ are used to model these two situations of short and long durations of postsynaptic action of DA, corresponding respectively to a unique go-signal delivery and to the combination of go-signal and reward. Solving the system of Equations 1 and 2, which describe the behavior of the network, is realized in the same time that performance is quantified, thereby determining whether the reward is delivered.

\section{Evaluation of Network Performance}

In order to evaluate the performance of the network, sustained activities are considered in the off state if during more than half of the time between two movements, activity is inferior or equal to 0.5. An error is counted each time that two successive stable states are repeated (e.g., on-on or off-off). For $n$ intervals between two movements, there are $n-1$ possible errors. The proportion of errors is the number of errors divided by $n-1$ possible errors, and the percentage of success is $100(1-k /(n-1))$. Initiation of the evaluation of a correct alternation is done by always rewarding the first two movements.

\section{Acknowledgments}

This work was supported by DRET no. 98 A0072 to J.-C. D. and was part of his PhD dissertation (January 1999, Paris). We thank Dr. A. Ishai for editing the manuscript.

Reprint requests should be sent to Jean-Claude Dreher, Clinical Brain Disorder Branch, NIMH, Room 4C108, MSC 1440, Bethesda, MD 20892-1440, USA, or via e-mail: dreherj@intra. nimh.nih.gov.

\section{REFERENCES}

Alexander, G. E. (1982). Functional development of frontal association cortex in monkeys: Behavioural and electrophysiological studies. Neurosciences Research Program Bulletin, 20, 471-479.

Arnsten, A. F. (1997). Catecholamine regulation of the prefrontal cortex. Journal of Psychopharmacology, 11, $151-162$.

Arnsten, A. F., \& Goldman-Rakic, P. S. (1998). Noise stress impairs prefrontal cortical cognitive function in monkeys: Evidence for a hyperdopaminergic mechanism. Archives of General Psychiatry, 55, 362-368.

Arnsten, A. F. T., Cai, L. X., Murphy, B. L., \& Goldman-Rakic, P. S. (1994). Dopamine D1 receptor mechanisms in the cognitive performance of young and aged monkeys. Psychopharmacology (Berlin), 116, 143-151.

Berger, B., Gaspar, P., \& Verney, C. (1991). Dopaminergic innervation of the cerebral cortex: Unexpected differences between rodents and primates. Trends in Neurosciences, 14, 21-27.

Braver, T. S., Barch, D. M., \& Cohen, J. D. (1999). Cognition and control in schizophrenia: A computational model of dopamine and prefrontal function. Biological Psychiatry, 46, 312-328.

Brito, G. N., \& Brito, L. S. (1990). Septohippocampal system and the prelimbic sector of frontal cortex: A neuropsychological battery analysis in the rat. Behavioural Brain Research, 36, 127-146.

Brozoski, T. J., Brown, R. M., Rosvold, H. E., \& Goldman, P. S. (1979). Cognitive deficit caused by regional depletion of dopamine in prefrontal cortex of rhesus monkey. Science, 205, 929-932.

Bubser, M., \& Schmidt, W. J. (1990). 6-Hydroxydopamine lesion of the rat prefrontal cortex increases locomotor activity, impairs acquisition of delayed alternation tasks, but does not affect uninterrupted tasks in the radial maze. Behavioural Brain Research, 37, 157-168. 
Cai, J. X., \& Arnsten, A. F. (1997). Dose-dependent effects of the dopamine D1 receptor agonists A77636 or SKF81297 on spatial working memory in aged monkeys. Journal of Pharmacology and Experimental Therapeutics, 283, 183-189

Camperi, M., \& Wang, X. J. (1998). A model of visuospatial working memory in prefrontal cortex: Recurrent network and cellular bistability. Journal of Computational Neuroscience, 5, 383-405.

Carlson, S., Rama, P., Tanila, H., Linnankoski, I., \& Mansikka, H. (1997). Dissociation of mnemonic coding and other functional neuronal processing in the monkey prefrontal cortex. Journal of Neurophysiology, 77, 761-774.

Cepeda, C., Radisavljevic, Z., Peacock, W., Levine, M. S., \& Buchwald, N. A. (1992). Differential modulation by dopamine of responses evoked by excitatory amino acids in human cortex. Synapse, 11, 330-341.

Cohen, J. D., Braver, T. S., \& O'Reilly, R. C. (1996). A computational approach to prefrontal cortex, cognitive control and schizophrenia: Recent developments and current challenges. Philosophical Transactions of the Royal Society of London, Series B: Biological Sciences, 351, 1515-1527.

Cohen, J. D., \& Servan-Schreiber, D. (1992). Context, cortex, and dopamine: A connectionist approach to behavior and biology in schizophrenia. Psychological Review, 99, 45-77.

Connors, B. W., Gutnick, M. J., \& Prince, D. A. (1982). Electrophysiological properties of neocortical neurons in vitro. Journal of Neurophysiology, 48, 1302-1320.

Constantinidis, C., \& Steinmetz, M. A. (1996). Neuronal activity in posterior parietal area 7 a during the delay periods of a spatial memory task. Journal of Neurophysiology, 76, $1352-1355$.

Contreras-Vidal, J. L., \& Schultz, W. (1999). A predictive reinforcement model of dopamine neurons for learning approach behavior. Journal of Computational Neuroscience, 6, 191-214.

Delatour, B., \& Gisquet-Verrier, P. (1996). Prelimbic cortex specific lesions disrupt delayed-variable response tasks in the rat. Behavioral Neuroscience, 110, 1282-1298.

Delord, B., Klaassen, A. J., Burnod, Y., Costalat, R., \& Guigon, E. (1997). Bistable behaviour in a neocortical neurone model. NeuroReport, 8, 1019-1023.

Delord, B., Klaassen, A. J., Burnod, Y., \& Guigon, E. (1996). An intrinsic bistable mechanism in neocortical pyramidal neurons might be involved in the generation of sustained discharge patterns related to working memory. Neural Network World, 4, 525-533.

Deutch, A. Y., Clark, W. A., \& Roth, R. H. (1990). Prefrontal cortical dopamine depletion enhances the responsiveness of mesolimbic dopamine neurons to stress. Brain Research, 25, 311-315.

di Pellegrino, G., \& Wise, S. P. (1993). Visuospatial versus visuomotor activity in the premotor and prefrontal cortex of a primate. Journal of Neuroscience, 13, 1227-1243.

Durstewitz, D., Kelc, M., \& Gunturkun, O. (1999). A neurocomputational theory of the dopaminergic modulation of working memory functions. Journal of Neuroscience, 19, 2807-2822.

Durstewitz, D., Seamans, J. K., \& Sejnowski, T. J. (2000). Dopamine-mediated stabilization of delay-period activity in a network model of prefrontal cortex. Journal of Neurophysiology, 83, 1733-1750.

Ferron, A., Thierry, A. M., Le Douarin, C., \& Glowinski, J. (1984). Inhibitory influence of the mesocortical dopaminergic system on spontaneous activity or excitatory response induced from the thalamic mediodorsal nucleus in the rat medial prefrontal cortex. Brain Research, 302, $257-265$.

Friston, K. J., Tononi, G., Reeke, G. N. J., Sporns, O., \& Edelman, G. M. (1994). Value-dependent selection in the brain: simulation in a synthetic neural model. Neuroscience, 59, 229-243.

Funahashi, S., Bruce, C. J., \& Goldman-Rakic, P. S. (1989). Mnemonic coding of visual space in the monkey's dorsolateral prefrontal cortex. Journal of Neurophysiology, 61, 331-349.

Fuster, J. M. (1973). Unit activity in prefrontal cortex during delayed-response performance: Neuronal correlates of transient memory. Journal of Neurophysiology, 36, 61-78.

Fuster, J. M. (1995). Memory in the cerebral cortex-An empiracal approach to neural networks in the buman and nonbuman primates. Cambridge: MIT Press.

Fuster, J. M. (2001). The prefrontal cortex-An update. Time is of the essence. Neuron, 30, 319-333.

Gao, W. J., Krimer, L. S., \& Goldman-Rakic, P. S. (2001). Presynaptic regulation of recurrent excitation by D1 receptors in prefrontal circuits. Proceedings of the National Academy of Sciences, U.S.A., 2, 295-300.

Garris, P. A., Christensen, J. R., Rebec, G. V., \& Wightman, R. M. (1997). Real-time measurement of electrically evoked extracellular dopamine in the striatum of freely moving rats. Journal of Neurochemistry, 68, 152-161.

Garris, P. A., Ciolkowski, E. L., Pastore, P., \& Wightman, R. M. (1994). Efflux of dopamine from the synaptic cleft in the nucleus accumbens of the rat brain. Journal of Neuroscience, 14, 6084-6093.

Godbout, R., Mantz, J., Pirot, S., Glowinski, J., \& Thierry, A. M. (1991). Inhibitory influence of the mesocortical dopaminergic neurons on their target cells: Electrophysiological and pharmacological characterization. Journal of Pharmacological and Experimental Therapeutics, 258, 728-738.

Goldman-Rakic, P. S. (1995). Cellular basis of working memory. Neuron, 14, 477-485.

Goldman-Rakic, P. S., Muly, E. C., \& Williams, G. V. (2000). D(1) receptors in prefrontal cells and circuits. Brain Research Brain Research Reviews, 31, 295-301.

Gonon, F. (1997). Prolonged and extrasynaptic excitatory action of dopamine mediated by D1 receptors in the rat striatum in vivo. Journal of Neuroscience, 17, 5972-5978.

Gorelova, N., \& Yang, C. R. (1997). The course of neural projection from the prefrontal cortex to the nucleus accumbens in the rat. Neuroscience, 76, 689-706.

Gulledge, A. T., \& Jaffe, D. B. (2001). Multiple effects of dopamine on layer $\mathrm{V}$ pyramidal cell excitability in rat prefrontal cortex. Journal of Neurophysiology, 86, 586-595.

Hammond, C., \& Crépel, V. (1992). Evidence for a slowly inactivating $\mathrm{K}+$ current in prefrontal cortical cells. European Journal of Neuroscience, 4, 1087-1092.

Hasegawa, R. P., Blitz, A. M., Geller, N. L., \& Goldberg, M. E. (2000). Neurons in monkey prefrontal cortex that track past or predict future performance. Science, 290, 1786-1789.

Hille, B. (1992). G protein-coupled mechanisms and nervous signaling. Neuron, 9, 187-195.

Jacobsen, C. F. (1935). Functions of frontal association areas in primates. Archives of Neurology and Psychiatry, 33, $558-569$.

Jay, T. M., Glowinski, J., \& Thierry, A. M. (1995). Inhibition of hippocampoprefrontal cortex excitatory responses by the mesocortical DA system. NeuroReport, 6, 1845-1848.

Jedema, H. P., \& Moghddam, B. (1996). Characterization of excitatory amino acid modulation of dopamine release in the prefrontal cortex of conscious rats. Journal of Neurochemistry, 66, 1448-1453. 
Jones, E. G. (1984). Laminar distribution of cortical efferent cells. In A. Peters \& E. Jones (Eds.), Cerebral cortex, (vol. 1, pp. 521-552). New York: Plenum.

Joyce, J. N. (1993). The dopamine hypothesis of schizophrenia: Limbic interactions with serotonin and norepinephrine. Psychopharmacology (Berlin), 112, 16-34.

Kritzer, M. F., \& Goldman-Rakic, P. S. (1995). Intrinsic circuit organization of the major layers and sublayers of the dorsolateral prefrontal cortex in the rhesus monkey. Journal of Comparative Neurology, 359, 131-143.

Kuroda, M., Murakami, K., Kishi, K., \& Price, J. L. (1995). Thalamocortical synapses between axons from the mediodorsal thalamic nucleus and pyramidal cells in the prelimbic cortex of the rat. Journal of Comparative Neurology, 356, 143-151.

Leon, M. I., \& Shadlen, M. N. (1999). Effect of expected reward magnitude on the response of neurons in the dorsolateral prefrontal cortex of the macaque. Neuron, 24, 415-425.

Levitt, J. B., Lewis, D. A., Yoshioka, T., \& Lund, J. S. (1993). Topography of pyramidal neuron intrinsic connections in macaque monkey prefrontal cortex (areas 9 and 46). Journal of Comparative Neurology, 338, 360-376.

Lewis, B. L., \& O’Donnell, P. (2000). Ventral tegmental area afferents to the prefrontal cortex maintain membrane potential "up" states in pyramidal neurons via $\mathrm{D}(1)$ dopamine receptors. Cerebral Cortex, 10, 1168-1175.

Lidow, M. S., Goldman-Rakic, P. S., Gallager, D. W., \& Rakic, P. (1991). Distribution of dopaminergic receptors in the primate cerebral cortex: Quantitative autoradiographic analysis using $[3 \mathrm{H}]$ raclopride, $[3 \mathrm{H}]$ spiperone and $[3 \mathrm{H}] \mathrm{SCH}$ 23390. Neuroscience, 40, 657-671.

Ljungberg, T., Apicella, P., \& Schultz, W. (1991). Responses of monkey midbrain dopamine neurons during delayed alternation performance. Brain Research, 567, 337-341.

Marder, E., Abbott, L. F., Turrigiano, G. G., \& Golowasch, J. (1996). Memory from the dynamics of intrinsic membrane currents. Proceedings of the National Academy of Sciences, U.S.A., 93, 13481-13486.

Martinot, J. L., Paillere-Martinot, M. L., Poirier, M. F., Feline, A., Allilaire, J. F., Dao-Castellana, M. H., Loc'h, C., Maziere, B., \& Syrota, A. (1996). In vivo characteristics of dopamine D2 receptor occupancy by amisulpride in schizophrenia. Psychopharmacology, 124, 154-158.

Maurice, N., Tkatch, T., Meisler, M., Sprunger, L. K., \& Surmeier, D. J. (2001). D1/D5 dopamine receptor activation differentially modulates rapidly inactivating and persistent sodium currents in prefrontal cortex pyramidal neurons. Journal of Neuroscience, 21, 2268-2277.

Miller, E. K., \& Cohen, J. D. (2001). An integrative theory of prefrontal cortex function. Annual Review of Neuroscience, 24, 167-202.

Miller, E. K., Erickson, C. A., \& Desimone, R. (1996a). Neural mechanisms of visual working memory in prefrontal cortex of the macaque. Journal of Neuroscience, 16, 5154-5167.

Miller, E. K., Erickson, C. A., \& Desimone, R. (1996b). Neural mechanisms of visual working memory in prefrontal cortex of the macaque. Journal of Neuroscience, 16, 5154-5167.

Montague, P. R., Dayan, P., \& Sejnowski, T. J. (1996). A framework for mesencephalic dopamine systems based on predictive Hebbian learning. Journal of Neuroscience, 16, 1936-1947.

Muller, U., von Cramon, D. Y., \& Pollmann, S. (1998). D1- versus D2-receptor modulation of visuospatial working memory in humans. Journal of Neuroscience, 18, $2720-2728$.

Murphy, B. L., Arnsten, A. F., Goldman-Rakic, P. S., \& Roth, R. H. (1996). Increased dopamine turnover in the prefrontal cortex impairs spatial working memory performance in rats and monkeys. Proceedings of the National Academy of Sciences, U.S.A., 93, 1325-1329.

Murphy, B. L., Roth, R. H., \& Arnsten, A. F. (1997). Clozapine reverses the spatial working memory deficits induced by FG7142 in monkeys. Neuropsychopharmacology, 16, 433-437.

Niki, H. (1974a). Differential activity of prefrontal units during right and left delayed response trials. Brain Research, 70, 346-349.

Niki, H. (1974b). Prefrontal unit activity during delayed alternation in the monkey: II. Relation to absolute versus relative direction of response. Brain Research, 68, 197-204.

Okubo, Y., Suhara, T., Suzuki, K., Kobayashi, K., Inoue, O., Terasaki, O., Someya, Y., Sassa, T., Sudo, Y., Matsushima, E., Iyo, M., Tateno, Y., \& Toru, M. (1997). Decreased prefrontal dopamine D1 receptors in schizophrenia revealed by PET. Nature, 385, 634-636.

Parsons, L. H., \& Justice, J. B., Jr. (1992). Extracellular concentration and in vivo recovery of dopamine in the nucleus accumbens using microdialysis. Journal of Neurochemistry, 58, 212-218.

Pirot, S., Godbout, R., Mantz, J., Tassin, J. P., Glowinski, J., \& Thierry, A. M. (1992). Inhibitory effects of ventral tegmental area stimulation on the activity of prefrontal cortical neurons: Evidence for the involvement of both dopaminergic and GABAergic components. Neuroscience, 49, 857-865.

Richardson, N. R., \& Gratton, A. (1998). Changes in medial prefrontal cortical dopamine levels associated with response-contingent food reward: An electrochemical study in rat. Journal of Neuroscience, 18, 9130-9138.

Sawaguchi, T., \& Goldman-Rakic, P. S. (1991). D1 dopamine receptors in prefrontal cortex: Involvement in working memory. Science, 251, 947-950.

Sawaguchi, T., \& Goldman-Rakic, P. S. (1994). The role of D1-dopamine receptor in working memory: Local injections of dopamine antagonists into the prefrontal cortex of rhesus monkeys performing an oculomotor delayed-response task. Journal of Neurophysiology, 71, 515-528.

Sawaguchi, T., Matsumura, M., \& Kubota, K. (1990). Catecholaminergic effects on neuronal activity related to a delayed response task in monkey prefrontal cortex. Journal of Neurophysiology, 63, 1385-1400.

Schultz, W. (1997). Dopamine neurons and their role in reward mechanisms. Current Opinion in Neurobiology, 7, 191-197.

Schultz, W., \& Dickinson, A. (2000). Neuronal coding of prediction errors. Annual Review of Neuroscience, 23, $473-500$.

Seamans, J. K., Durstewitz, D., Christie, B. R., Stevens, C. F., \& Sejnowski, T. J. (2001). Dopamine D1/D5 receptor modulation of excitatory synaptic inputs to layer $\mathrm{V}$ prefrontal cortex neurons. Proceedings of the National Academy of Sciences, U.S.A., 98, 301-306.

Seamans, J. K., Gorelova, N., Durstewitz, D., \& Yang, C. R. (2001). Bidirectional dopamine modulation of GABAergic inhibition in prefrontal cortical pyramidal neurons. Journal of Neuroscience, 21, 3628-3638.

Servan-Schreiber, D., Printz, H., \& Cohen, J. D. (1990). A network model of catecholamine effects: Gain, signal-to-noise ratio, and behavior. Science, 249, 892-895.

Silva, L. R., Amitai, Y., \& Connors, B. W. (1991). Intrinsic oscillations of neocortex generated by layer 5 pyramidal neurons. Science, 251, 432-435.

Simon, H. (1981). Dopaminergic A10 neurons and frontal system. Journal of Physiology (Paris), 77, 81-95.

Smiley, J. F., Levey, A. I., Ciliax, B. J., \& Goldman-Rakic, P. S. (1994). D1 dopamine receptor immunoreactivity in human and monkey cerebral cortex: Predominant and extrasynaptic 
localization in dendritic spines. Proceedings of the National Academy of Sciences, U.S.A., 91, 5720-5724.

Suri, R. E., \& Schultz, W. (2001). Temporal difference model reproduces anticipatory neural activity. Neural Computation, 13, 841-862.

Suzuki, W. A., Miller, E. K., \& Desimone, R. (1997). Object and place memory in the macaque entorhinal cortex. Journal of Neurophysiology, 78, 1062-1081.

Taber, M. T., Das, S., \& Fibiger, H. C. (1995). Cortical regulation of subcortical dopamine release: Mediation via the ventral tegmental area. Journal of Neurochemistry, 65, 1407-1410.

Thorpe, S., Fize, D., \& Marlot, C. (1996). Speed of processing in the human visual system. Nature, 6, 520-522.

Tremblay, L., \& Schultz, W. (1999). Relative reward preference in primate orbitofrontal cortex. Nature, 398, 704-708.

Waelti, P., Dickinson, A., \& Schultz, W. (2001). Dopamine responses comply with basic assumptions of formal learning theory. Nature, 412, 43-48.

Wang, J., \& O'Donnell, P. (2001). D(1) dopamine receptors potentiate nmda-mediated excitability increase in layer $\mathrm{V}$ prefrontal cortical pyramidal neurons. Cerebral Cortex, 11, $452-462$.
Watanabe, M. (1996). Reward expectancy in primate prefrontal neurons. Nature, 382, 629-632.

Watanabe, M., Kodama, T., \& Hikosaka, K. (1997). Increase of extracellular dopamine in primate prefrontal cortex during a working memory task. Journal of Neurophysiology, 78, 2795-2798.

Williams, G. V., \& Goldman-Rakic, P. S. (1995). Modulation of memory fields by dopamine D1 receptors in prefrontal cortex. Nature, 376, 572-575.

Wortwein, G., Mogensen, J., \& Divac, I. (1994). Retention and relearning of spatial delayed alternation in rats after ablation of the prefrontal or total non-prefrontal isocortex. Behavioural Brain Research, 63, 127-131.

Yang, C. R., \& Seamans, J. K. (1996). Dopamine D1 receptor actions in layers V-VI rat prefrontal cortex neurons in vitro: Modulation of dendritic-somatic signal integration. Journal of Neuroscience, 16, 1922-1935.

Zahrt, J., Taylor, J. R., Mathew, R. G., \& Arnsten, A. F. (1997). Supranormal stimulation of D1 dopamine receptors in the rodent prefrontal cortex impairs spatial working memory performance. Journal of Neuroscience, 17 , $8528-8535$. 